Revue internationale de l'économie sociale

Recma

\title{
Le monde coopératif dans une économie plurielle : le problème
} de l'attractivité

\section{Cooperatives in a plural Economy: The Issue of their Attractiveness}

\section{Jérôme Blanc et Denis Colongo}

Numéro 320, avril 2011

Le monde coopératif dans une économie plurielle : le problème de l'attractivité

Cooperatives in a plural Economy: The issue of their Attractiveness

URI : https://id.erudit.org/iderudit/1020905ar

DOI : https://doi.org/10.7202/1020905ar

Aller au sommaire du numéro

Éditeur(s)

Association Recma

ISSN

1626-1682 (imprimé)

2261-2599 (numérique)

Découvrir la revue

Citer ce document

Blanc, J. \& Colongo, D. (2011). Le monde coopératif dans une économie plurielle : le problème de l'attractivité. Revue internationale de l'économie sociale, (320), 24-25. https://doi.org/10.7202/1020905ar 


\section{Dossier}

\section{LE MONDE COOPÉRATIF DANS UNE ÉCONOMIE PLURIELLE: LE PROBLĖME DE L'ATTRACTIVITÉ}

\begin{abstract}
* Maître de conférences HDR, université Lumière-Lyon 2 et chercheur au laboratoire Triangle UMR 5206. Mél.: jerome.blanc@ univ-lyon2.fr.

**Professeur associé à l'université Lumière-Lyon 2, secrétaire général de Cress Rhône-Alpes. Mél.: dcolongo@cress-rhone-alpes.org.
\end{abstract}

(1) Organisé par un partenariat entre le Lefi (Laboratoire d'économie de la firme et des institutions, désormais inclus dans I'UMR Triangle) et la Cress Rhône-Alpes, il a précédé de peu l'ouverture de la chaire d'entrepreneuriat en ESS à l'université Lumière-Lyon 2. II a été soutenu par la Fondation du Crédit coopératif, le Crédit mutuel, la CGScop, La NEF, la Caisse d'épargne Rhône-Alpes, le Collège coopératif Rhône-Alpes, la ville de Lyon, le conseil régiona Rhône-Alpes et le Grand Lyon.

(2) L'appel à communications, le programme ainsi que la liste des textes présentés sont disponibles sur www.cress-rhone-alpes. org/cress/rubrique.php3?id rubrique=122.

\author{
par Jérôme Blanc* et Denis Colongo**
}

$\mathrm{L}$ e dossier qui suit est constitué d'une sélection de quatre textes parmi les soixante-trois présentés lors du dernier colloque européen du comité de recherche de l'Alliance coopérative internationale (ACI), qui s'est tenu à l'université Lumière-Lyon 2 les 2, 3 et 4 septembre $2010^{(1)}$. Lappel à communications a concentré les débats sur le thème des contributions des coopératives à une économie plurielle (2).

Trois grands secteurs ou types d'activité ont été particulièrement l'objet de travaux: les coopératives dans la banque et les services financiers, les coopératives agricoles et dans le monde rural et les coopératives de production. Par ailleurs, trois ensembles de questions ont traversé les débats: sur les règles européennes et nationales en matière de concurrence, d'aides publiques et de fiscalité, sur la mise en œuvre du statut de société coopérative européenne et sur les contributions des coopératives au développement local. Finalement, la question de la pluralité a largement renvoyé à celle de l'attractivité du monde coopératif, qui entremêle plusieurs dimensions de l'articulation des coopératives avec leur environnement et qui est ainsi la question centrale de la pluralité économique vue dans une perspective dynamique. C'est sur ce point à la fois central et transversal que cette courte présentation met l'accent.

\section{L'importance de la loi}

L'attractivité des coopératives renvoie d'abord à des questions statutaires. La loi fournit les conditions dans lesquelles s'inscrit l'action coopérative et façonne donc largement la nature des dynamiques coopératives. Les cas discutés durant le colloque ont montré à ce titre un paysage contrasté. Il en ressort principalement que, si les lois portant statuts sont essentielles, les lois et règlements qui concernent les activités elles-mêmes sont tout aussi importantes et peuvent représenter une contrainte affaiblissant la position des coopératives. Dans le dossier qui suit, deux textes alimentent ce débat: celui de Franci Avsec et Primož Žerjav portant sur les effets du statut de société coopérative européenne en Slovénie et celui de Jan Myers, Molly Scott-Cato et Paul A. Jones portant sur les contraintes que les politiques publiques d'inclusion financière font peser sur les coopératives 
de crédit au pays de Galles. Ce dernier cas met aussi l'accent sur les politiques publiques. Celles-ci ont bien souvent un rôle déterminant. Il suffit pour s'en convaincre d'évoquer en France le poids des pouvoirs publics dans le rapprochement de deux banques pourtant coopératives, les Banques populaires et les Caisses d'épargne. Yifat Solel présente un cas extrême de détermination politique de l'évolution d'activités coopératives, avec la démutualisation des coopératives de transport en Israël, derrière le prétexte de la privatisation pour des raisons d'efficacité.

La fiscalité et les soutiens publics éventuels constituent un autre ensemble d'éléments influençant le degré d'attractivité du monde coopératif. En la matière, le traitement spécifique des coopératives se heurte aux règles européennes relatives à la concurrence et aux aides d'Etat, de même qu'à certaines dispositions fiscales nationales, qui rendent plus difficiles la possibilité pour le monde coopératif de bénéficier de politiques publiques favorables. Les nombreux juristes qui ont discuté de ces questions lors du colloque n'ont cependant pas conclu à l'impossibilité et travaillent au contraire dans le sens de l'identification des conditions dans lesquelles des avantages fiscaux et des soutiens publics demeurent justifiables: les règles européennes ne sont pas figées et font preuve d'un certain degré de souplesse, elles nécessitent des clarifications, mais il reste à poser les fondements doctrinaux aptes à justifier ces soutiens.

\section{Adaptations coopératives}

D'un point de vue théorique, l'attractivité du monde coopératif pourrait se résumer dans son efficience relativement aux organisations non coopératives; pourtant, comme l'ont montré plusieurs communications, la notion d'efficience est biaisée par son absence de prise en compte d'externalités et son usage vient en conséquence trop souvent à charge contre les formes plurielles d'organisation de la production, dont les coopératives. Dans ce dossier, Roger Spear souligne que la pluralité s'exprime dans les organisations elles-mêmes: les coopératives ne sont pas exemptes de transformations provenant de dynamiques internes ou contextuelles qui complexifient la donne. Alors que certaines adaptations permettent de rester dans le cadre des principes coopératifs, d'autres constituent des hybridations où l'isomorphisme, poussé par la pression concurrentielle, l'emporte sur ces principes.

Au fond, les coopératives participent à la pluralité économique de plusieurs façons. D'abord, elles rompent la dualité lucratif-public; ensuite, elles sont elles-mêmes plurielles du fait de la grande variété de leurs formes, que vient renforcer une dynamique continue d'innovations organisationnelles; enfin, elles sont fréquemment partie prenante de dynamiques de développement local où une pluralité d'acteurs intervient dans des objectifs eux-mêmes pluriels et irréductibles au seul motif de profit. 